\title{
Diastereoselective Synthesis of Fulleropyrrolidines from Suitably Functionalized Chiral Cyclobutanes
}

\author{
Beatriz M. Illescas, Nazario Martín* \\ Departamento de Química Orgánica, Facultad de Química, Universidad Complutense, E- \\ 28040 Madrid, Spain \\ Jordi Poater \\ Afdeling Theoretische Chemie, Scheikundig Laboratorium der Vrije Universiteit, De \\ Boelelaan 1083, NL-1081 HV Amsterdam, The Netherlands. \\ Miquel Solà \\ Institut de Química Computacional and Departament de Química, Universitat de Girona, \\ Campus de Montilivi, 17071 Girona, Catalonia, Spain. \\ Gemma P. Aguado, Rosa M. Ortuño \\ Departament de Química, Universitat Autònoma de Barcelona, 08193 Bellaterra, Barcelona, \\ Spain \\ nazmar@quim.ucm.es
}

$\begin{array}{ll}\text { Table of Contents } & \text { S1 }\end{array}$

$\begin{array}{lr}\text { Computational Details } & \text { S2 }\end{array}$

BP86 optimized xyz coordinates of the cis and trans isomers of compound 9

${ }^{1}$ H NMR Spectra $\quad$ S7-S9

$\begin{array}{lr}\text { References } & \text { S10 }\end{array}$ 


\section{Computational Details}

The reported calculations have been carried out by using the 2004.01 release of the Amsterdam density functional (ADF) ${ }^{1}$ package developed by Baerends $e$ t $a l^{2,3}$ The numerical integration scheme employed has been that of te Velde and Baerends. ${ }^{4}$ Both geometry optimizations, energy evaluations, and nuclear spin-spin coupling constants (J) of neutral closed-shell singlet ground-state structures has been performed using a generalized gradient approximation (GGA) that includes the GGA exchange correction of Becke $\mathrm{e}^{5}$ and the GGA correlation correction of Perdew, ${ }^{6}$ giving the so-called BP86 functional. For geometry optimizations we have used an uncontracted double- $\varsigma$ basis set augmented by an extra polarization function (DZP basis set) for describing the $2 \mathrm{~s}$ and $2 \mathrm{p}$ orbitals of carbon, nitrogen, and oxygen, and the $1 \mathrm{~s}$ orbital of hydrogen. ${ }^{7,8}$ The calculation of the $\mathrm{J}$ constants has been carried out with the CPL code ${ }^{9}$ of the ADF2004.01 program at the BP86/DZP optimized geometries using an uncontracted triple- $\varsigma$ basis set augmented by two extra polarizations functions for describing the $2 \mathrm{~s}$ and $2 \mathrm{p}$ orbitals of carbon, nitrogen, and oxygen, and the $1 \mathrm{~s}$ orbital of hydrogen ${ }^{7,8}$ The use of a triple zeta with polarization functions is recommended since large and flexible basis sets are necessary to obtain reliable values of J constants. ${ }^{9}$ The four terms contributing to the $\mathbf{J}$ constants have been taken into account in the BP86/TZ2P//BP86/DZP calculations: the paramagnetic and diamagnetic orbital terms, and the electron-spin dependent Fermi-contact and spin-dipole term. ${ }^{9}$ In all cases, the $1 \mathrm{~s}$ electrons of carbon, nitrogen, and oxygen were treated within the frozen core approximation. ${ }^{2}$ A set of auxiliary s, p, d, f, and g functions, centered in all nuclei, was introduced in order to fit the molecular density and Coulomb potential accurately in each SCF cycle. ${ }^{10}$ 
Table S1. xyz coordinates of the cis and trans isomers of compound 9 optimized at the BP86/DZP level of theory.

\begin{tabular}{|c|c|c|c|}
\hline $\mathrm{C}$ & 0.205802 & 2.489465 & -1.464339 \\
\hline $\mathrm{C}$ & -0.127294 & 3.055885 & -0.149422 \\
\hline $\mathrm{C}$ & 0.452325 & 2.543106 & 0.992009 \\
\hline $\mathrm{C}$ & 1.629104 & 1.570757 & 0.998678 \\
\hline $\mathrm{C}$ & 2.003995 & 0.967611 & -0.427904 \\
\hline $\mathrm{C}$ & 1.093471 & 1.437695 & -1.559641 \\
\hline $\mathrm{C}$ & -0.996168 & 2.504629 & -2.264188 \\
\hline $\mathrm{C}$ & -2.064451 & 3.069261 & -1.465940 \\
\hline $\mathrm{C}$ & -1.527175 & 3.408684 & -0.165056 \\
\hline $\mathrm{C}$ & -2.314007 & 3.241940 & 0.978949 \\
\hline $\mathrm{C}$ & -0.365696 & 2.332633 & 2.154551 \\
\hline $\mathrm{C}$ & 1.135874 & 0.556350 & 2.018446 \\
\hline $\mathrm{C}$ & 1.234250 & -0.808929 & 1.856688 \\
\hline $\mathrm{C}$ & 1.569879 & -1.373370 & 0.538613 \\
\hline $\mathrm{C}$ & 1.806508 & -0.542595 & -0.538538 \\
\hline $\mathrm{C}$ & 1.230109 & -0.876483 & -1.812068 \\
\hline $\mathrm{C}$ & 0.796952 & 0.349094 & -2.446931 \\
\hline $\mathrm{C}$ & -0.349921 & 0.358344 & -3.248798 \\
\hline $\mathrm{C}$ & -1.269766 & 1.462170 & -3.155231 \\
\hline $\mathrm{C}$ & -3.363044 & 2.574042 & -1.580626 \\
\hline $\mathrm{C}$ & -3.642090 & 1.482253 & -2.493071 \\
\hline $\mathrm{C}$ & -2.613565 & 0.937643 & -3.264921 \\
\hline $\mathrm{C}$ & -2.520267 & -0.499926 & -3.432920 \\
\hline $\mathrm{C}$ & -1.117037 & -0.859163 & -3.424640 \\
\hline $\mathrm{C}$ & -0.701922 & -2.039760 & -2.811075 \\
\hline $\mathrm{C}$ & 0.498484 & -2.054300 & -1.997534 \\
\hline $\mathrm{C}$ & 0.273029 & -2.928339 & -0.875926 \\
\hline $\mathrm{C}$ & 0.792260 & -2.580431 & 0.374933 \\
\hline $\mathrm{C}$ & 0.061439 & 1.105265 & 2.790665 \\
\hline $\mathrm{C}$ & -2.372522 & -2.735241 & 2.265823 \\
\hline $\mathrm{C}$ & -2.111395 & -1.682742 & 3.144916 \\
\hline $\mathrm{C}$ & -3.043886 & -0.575856 & 3.244743 \\
\hline $\mathrm{C}$ & -4.198897 & -0.564266 & 2.461994 \\
\hline $\mathrm{C}$ & -4.468078 & -1.658300 & 1.552548 \\
\hline $\mathrm{C}$ & -3.243702 & -3.283011 & 0.159086 \\
\hline $\mathrm{C}$ & -1.842694 & -3.639307 & 0.165443 \\
\hline $\mathrm{C}$ & -1.300674 & -3.294389 & 1.465087 \\
\hline $\mathrm{C}$ & -0.011931 & -2.770008 & 1.564346 \\
\hline $\mathrm{C}$ & 0.261584 & -1.679025 & 2.475349 \\
\hline $\mathrm{C}$ & -0.771181 & -1.149713 & 3.257103 \\
\hline $\mathrm{C}$ & -2.275640 & 0.639661 & 3.417506 \\
\hline $\mathrm{C}$ & -2.689152 & 1.821017 & 2.803185 \\
\hline $\mathrm{C}$ & -3.889818 & 1.835145 & 1.993010 \\
\hline $\mathrm{C}$ & -4.630313 & 0.664605 & 1.824356 \\
\hline $\mathrm{C}$ & -5.165986 & 0.328239 & 0.521751 \\
\hline $\mathrm{C}$ & -5.066571 & -1.107425 & 0.353705 \\
\hline $\mathrm{C}$ & -4.751706 & -1.644722 & -0.894838 \\
\hline $\mathrm{C}$ & -3.825339 & -2.754300 & -0.994364 \\
\hline $\mathrm{C}$ & -1.068945 & -3.459087 & -0.982657 \\
\hline $\mathrm{C}$ & -1.673360 & -2.910083 & -2.181326 \\
\hline $\mathrm{C}$ & -3.025364 & -2.564415 & -2.186191 \\
\hline $\mathrm{C}$ & -3.457037 & -1.335693 & -2.823933 \\
\hline $\mathrm{C}$ & -4.523394 & -0.768050 & -2.025620 \\
\hline $\mathrm{C}$ & -4.613016 & 0.615496 & -1.864360 \\
\hline $\mathrm{C}$ & -4.940944 & 1.174595 & -0.565026 \\
\hline
\end{tabular}




\begin{tabular}{lrrr}
$\mathrm{C}$ & -4.172244 & 2.386560 & -0.392041 \\
$\mathrm{C}$ & -3.655885 & 2.714279 & 0.863286 \\
$\mathrm{C}$ & -0.872091 & 0.276861 & 3.422138 \\
$\mathrm{C}$ & -1.717755 & 2.691193 & 2.168393 \\
$\mathrm{C}$ & -3.571427 & -2.723916 & 1.458137 \\
$\mathrm{C}$ & 3.515051 & 1.481370 & -0.688491 \\
$\mathrm{H}$ & 3.540888 & 1.913399 & -1.703708 \\
$\mathrm{C}$ & 2.988989 & 2.233243 & 1.465013 \\
$\mathrm{~N}$ & 3.757662 & 2.546730 & 0.278270 \\
$\mathrm{H}$ & 3.510664 & 1.485572 & 2.080950 \\
$\mathrm{H}$ & 2.814305 & 3.125314 & 2.083090 \\
$\mathrm{C}$ & 3.554506 & 3.907649 & -0.234283 \\
$\mathrm{H}$ & 4.209135 & 4.063451 & -1.102876 \\
$\mathrm{H}$ & 2.514014 & 4.127181 & -0.536018 \\
$\mathrm{H}$ & 3.852551 & 4.626615 & 0.541808 \\
$\mathrm{C}$ & 4.589113 & 0.390719 & -0.674239 \\
$\mathrm{C}$ & 6.009738 & 0.888795 & -1.061450 \\
$\mathrm{C}$ & 5.179678 & -0.184665 & 0.647795 \\
$\mathrm{H}$ & 4.264979 & -0.425167 & -1.343173 \\
$\mathrm{C}$ & 6.611115 & 0.023631 & 0.079021 \\
$\mathrm{H}$ & 6.124258 & 1.963442 & -0.847715 \\
$\mathrm{H}$ & 6.361695 & 0.674855 & -2.084445 \\
$\mathrm{H}$ & 5.016993 & 0.471349 & 1.511547 \\
$\mathrm{H}$ & 4.899113 & -1.214848 & 0.915577 \\
$\mathrm{H}$ & 7.298716 & 0.563844 & 0.752806 \\
$\mathrm{C}$ & 7.294534 & -1.250868 & -0.398418 \\
$\mathrm{H}$ & 6.642535 & -1.840050 & -1.070280 \\
$\mathrm{C}$ & 9.534803 & -0.886009 & -0.109590 \\
$\mathrm{C}$ & 8.956295 & -1.626298 & 1.119648 \\
$\mathrm{H}$ & 10.430645 & -1.373682 & -0.525794 \\
$\mathrm{H}$ & 9.772906 & 0.167535 & 0.124970 \\
$\mathrm{H}$ & 9.545498 & -2.509924 & 1.410898 \\
$\mathrm{H}$ & 8.860414 & -0.953114 & 1.991226 \\
$\mathrm{O}$ & 7.664669 & -2.093249 & 0.689205 \\
$\mathrm{O}$ & 8.498865 & -0.965531 & -1.106792 \\
& & & \\
\hline & & &
\end{tabular}




\section{trans}

\begin{tabular}{|c|c|c|c|}
\hline $\mathrm{C}$ & -0.468678 & 1.613225 & 2.224575 \\
\hline $\mathrm{C}$ & 0.166327 & 2.704703 & 1.470769 \\
\hline $\mathrm{C}$ & -0.154013 & 2.914442 & 0.144744 \\
\hline C & -1.320295 & 2.240298 & -0.578496 \\
\hline $\mathrm{C}$ & -2.018352 & 1.065542 & 0.248511 \\
\hline $\mathrm{C}$ & -1.380916 & 0.785117 & 1.605211 \\
\hline C & 0.520960 & 1.066278 & 3.122476 \\
\hline $\mathrm{C}$ & 1.754557 & 1.801193 & 2.938318 \\
\hline C & 1.533797 & 2.809132 & 1.922336 \\
\hline C & 2.556329 & 3.126205 & 1.022890 \\
\hline $\mathrm{C}$ & 0.904730 & 3.195439 & -0.786666 \\
\hline C & -0.628320 & 1.807777 & -1.859554 \\
\hline C & -0.792214 & 0.569290 & -2.442085 \\
\hline $\mathrm{C}$ & -1.430173 & -0.521284 & -1.686003 \\
\hline $\mathrm{C}$ & -1.882401 & -0.309772 & -0.398520 \\
\hline C & -1.618901 & -1.306283 & 0.601606 \\
\hline $\mathrm{C}$ & -1.316007 & -0.629595 & 1.842914 \\
\hline $\mathrm{C}$ & -0.381963 & -1.175523 & 2.729924 \\
\hline C & 0.560657 & -0.306677 & 3.385746 \\
\hline $\mathrm{C}$ & 2.981484 & 1.143098 & 3.018909 \\
\hline $\mathrm{C}$ & 3.020567 & -0.283658 & 3.274901 \\
\hline C & 1.831776 & -0.993746 & 3.454356 \\
\hline $\mathrm{C}$ & 1.669181 & -2.297978 & 2.840714 \\
\hline C & 0.296420 & -2.411674 & 2.392855 \\
\hline C & 0.004164 & -3.063883 & 1.196002 \\
\hline $\mathrm{C}$ & -0.979578 & -2.508936 & 0.286517 \\
\hline C & -0.526276 & -2.733731 & -1.061697 \\
\hline C & -0.738969 & -1.744340 & -2.026370 \\
\hline $\mathrm{C}$ & 0.605992 & 2.515075 & -2.027971 \\
\hline $\mathrm{C}$ & 2.767679 & -1.371903 & -3.112459 \\
\hline C & 2.737365 & 0.002201 & -3.356802 \\
\hline $\mathrm{C}$ & 3.693545 & 0.874244 & -2.701386 \\
\hline $\mathrm{C}$ & 4.640691 & 0.339342 & -1.827613 \\
\hline C & 4.670970 & -1.086490 & -1.577435 \\
\hline $\mathrm{C}$ & 3.124185 & -3.003809 & -1.467353 \\
\hline C & 1.753297 & -3.115443 & -1.912433 \\
\hline C & 1.529124 & -2.102175 & -2.924249 \\
\hline $\mathrm{C}$ & 0.309144 & -1.427605 & -2.974039 \\
\hline C & 0.275084 & -0.003312 & -3.228748 \\
\hline C & 1.470452 & 0.697579 & -3.422919 \\
\hline $\mathrm{C}$ & 3.013031 & 2.107235 & -2.363301 \\
\hline C & 3.303076 & 2.760033 & -1.166123 \\
\hline $\mathrm{C}$ & 4.288891 & 2.207931 & -0.259658 \\
\hline $\mathrm{C}$ & 4.944300 & 1.019161 & -0.583198 \\
\hline C & 5.162177 & 0.012122 & 0.434410 \\
\hline C & 4.994138 & -1.289291 & -0.179804 \\
\hline $\mathrm{C}$ & 4.390860 & -2.325723 & 0.533402 \\
\hline $\mathrm{C}$ & 3.440756 & -3.201028 & -0.122420 \\
\hline C & 0.743495 & -3.424160 & -0.998940 \\
\hline $\mathrm{C}$ & 1.072460 & -3.630100 & 0.398656 \\
\hline $\mathrm{C}$ & 2.395599 & -3.519661 & 0.827750 \\
\hline $\mathrm{C}$ & 2.699717 & -2.840530 & 2.072321 \\
\hline $\mathrm{C}$ & 3.932173 & -2.102725 & 1.889502 \\
\hline C & 4.088544 & -0.848103 & 2.480922 \\
\hline $\mathrm{C}$ & 4.715864 & 0.230538 & 1.738687 \\
\hline $\mathrm{C}$ & 4.034919 & 1.460773 & 2.074521 \\
\hline C & 3.824933 & 2.434327 & 1.095811 \\
\hline $\mathrm{C}$ & 1.639267 & 1.989556 & -2.810775 \\
\hline $\mathrm{C}$ & 2.233013 & 3.324216 & -0.365915 \\
\hline C & 3.751653 & -1.925438 & -2.209548 \\
\hline
\end{tabular}




$\begin{array}{lrrr}\mathrm{C} & -3.541952 & 1.587239 & 0.427123 \\ \mathrm{H} & -3.850255 & 1.419006 & 1.473258 \\ \mathrm{C} & -2.540130 & 3.207557 & -0.890359 \\ \mathrm{~N} & -3.515264 & 3.016962 & 0.158579 \\ \mathrm{H} & -2.962603 & 2.896717 & -1.859666 \\ \mathrm{H} & -2.219581 & 4.255374 & -0.972863 \\ \mathrm{C} & -3.339728 & 3.882773 & 1.329406 \\ \mathrm{H} & -4.147513 & 3.680778 & 2.046186 \\ \mathrm{H} & -2.372293 & 3.760038 & 1.847616 \\ \mathrm{H} & -3.432084 & 4.930145 & 1.008931 \\ \mathrm{C} & -4.524137 & 0.855717 & -0.483057 \\ \mathrm{C} & -4.913536 & -0.589173 & -0.038949 \\ \mathrm{C} & -6.015461 & 1.298576 & -0.297730 \\ \mathrm{H} & -4.188191 & 0.905322 & -1.531611 \\ \mathrm{C} & -6.396221 & -0.148853 & 0.121182 \\ \mathrm{H} & -4.444858 & -0.921271 & 0.899907 \\ \mathrm{H} & -4.751668 & -1.372438 & -0.795076 \\ \mathrm{H} & -6.156328 & 2.063627 & 0.481893 \\ \mathrm{H} & -6.510528 & 1.654285 & -1.216179 \\ \mathrm{H} & -6.774879 & -0.224545 & 1.155030 \\ \mathrm{C} & -7.374117 & -0.843883 & -0.816814 \\ \mathrm{H} & -7.030040 & -0.777832 & -1.867342 \\ \mathrm{C} & -8.554126 & -2.265735 & 0.504096 \\ \mathrm{C} & -9.424139 & -1.023192 & 0.220468 \\ \mathrm{H} & -9.097818 & -3.215449 & 0.386044 \\ \mathrm{H} & -8.106849 & -2.229005 & 1.514858 \\ \mathrm{H} & -10.393246 & -1.277675 & -0.239961 \\ \mathrm{H} & -9.600487 & -0.428102 & 1.134078 \\ \mathrm{O} & -8.672717 & -0.255714 & -0.742189 \\ \mathrm{O} & -7.535383 & -2.222855 & -0.508774 \\ & & & \end{array}$


${ }^{1} \mathrm{H}$ NMR of $7 \mathbf{a}\left(\mathrm{CDCl}_{3}, 300 \mathrm{MHz}\right)$

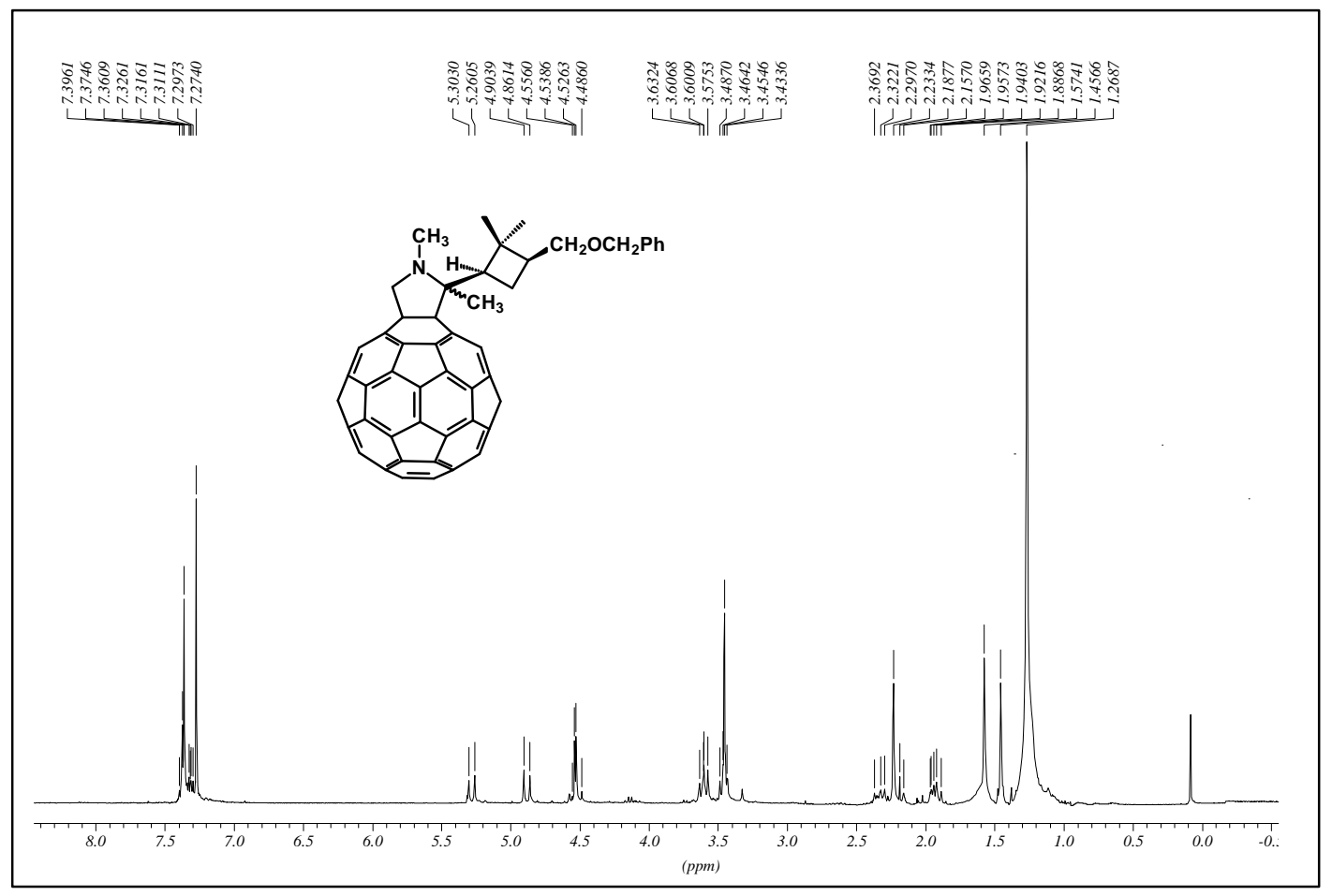

${ }^{13} \mathrm{C}$ NMR of $7 \mathbf{a}\left(\mathrm{CDCl}_{3}, 75 \mathrm{MHz}\right)$

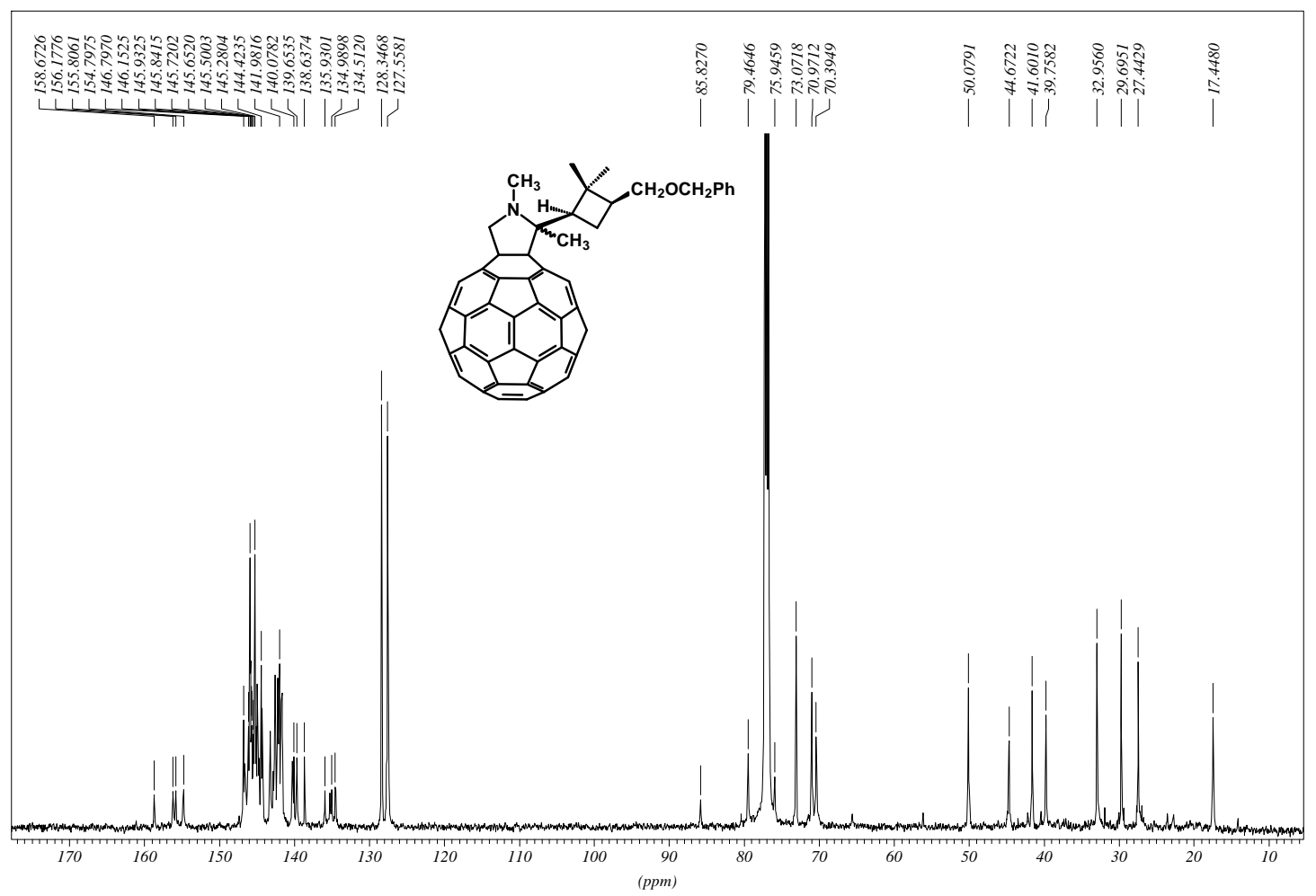


${ }^{1} \mathrm{H}$ NMR of $\mathbf{7 b}\left(\mathrm{CDCl}_{3}, 300 \mathrm{MHz}\right)$

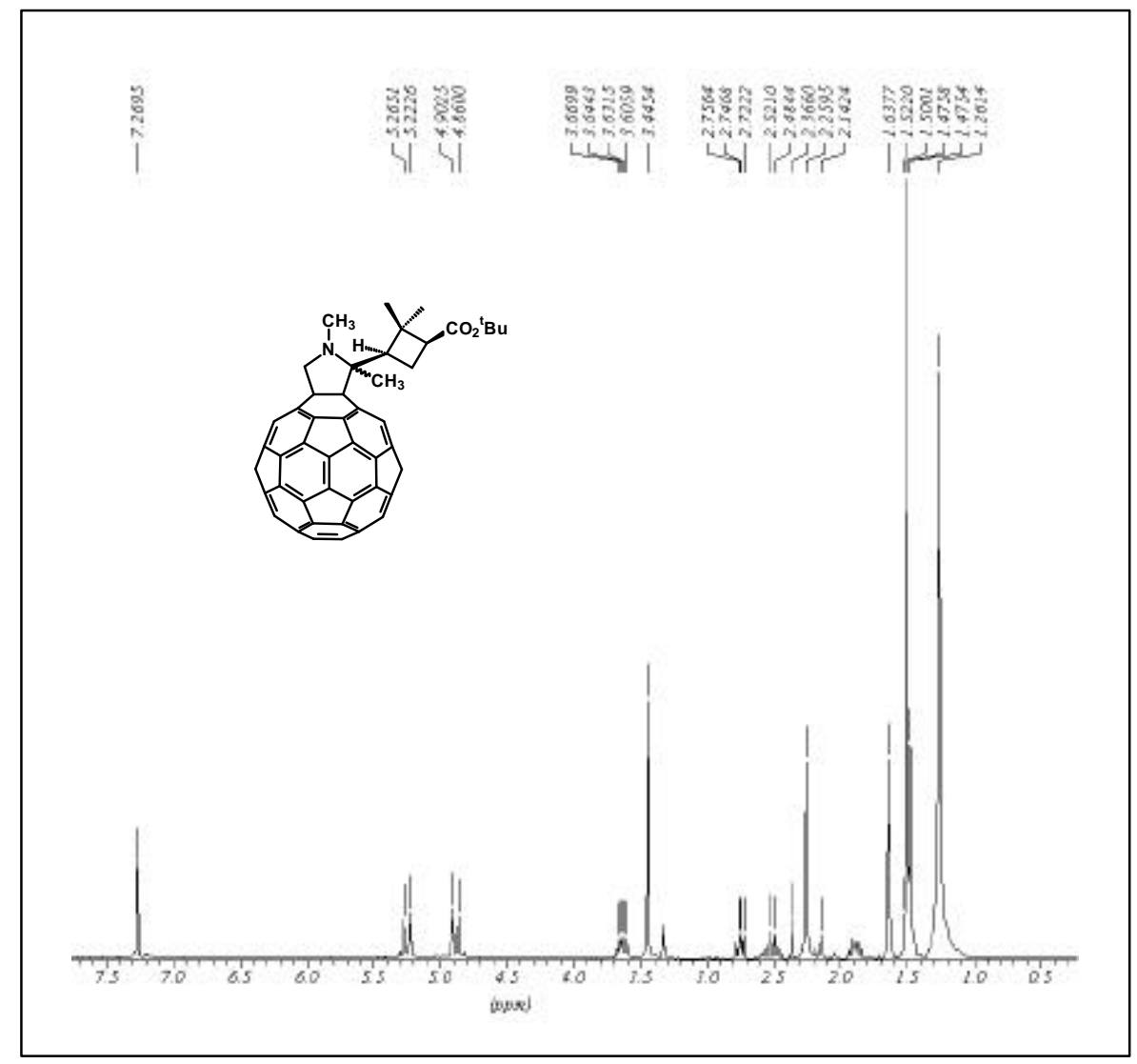


${ }^{1} \mathrm{H} \mathrm{NMR}$ of $9\left(\mathrm{CDCl}_{3}, 500 \mathrm{MHz}\right)$

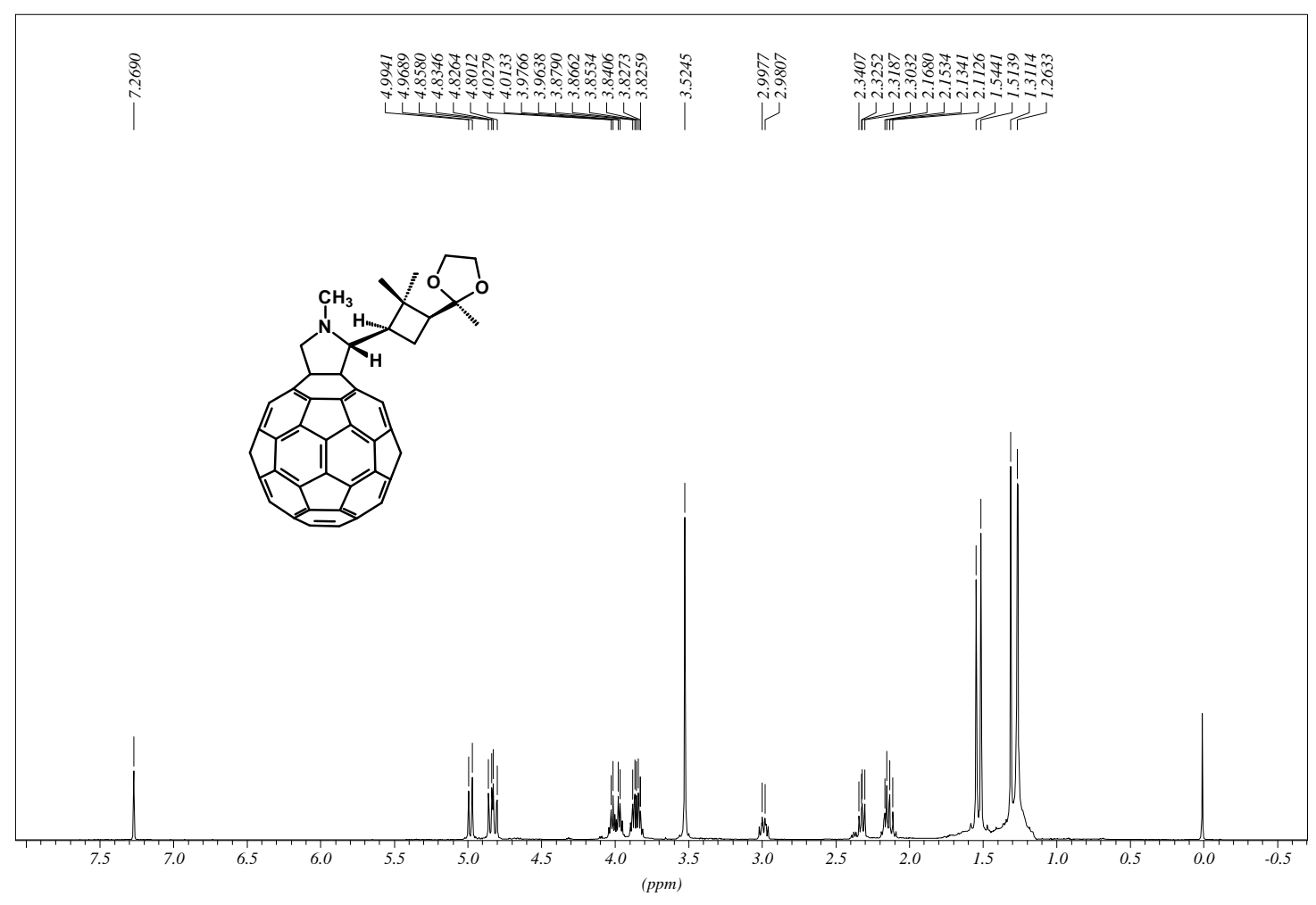

${ }^{13} \mathrm{C} \mathrm{NMR}$ of $9\left(\mathrm{CDCl}_{3}, 125 \mathrm{MHz}\right)$

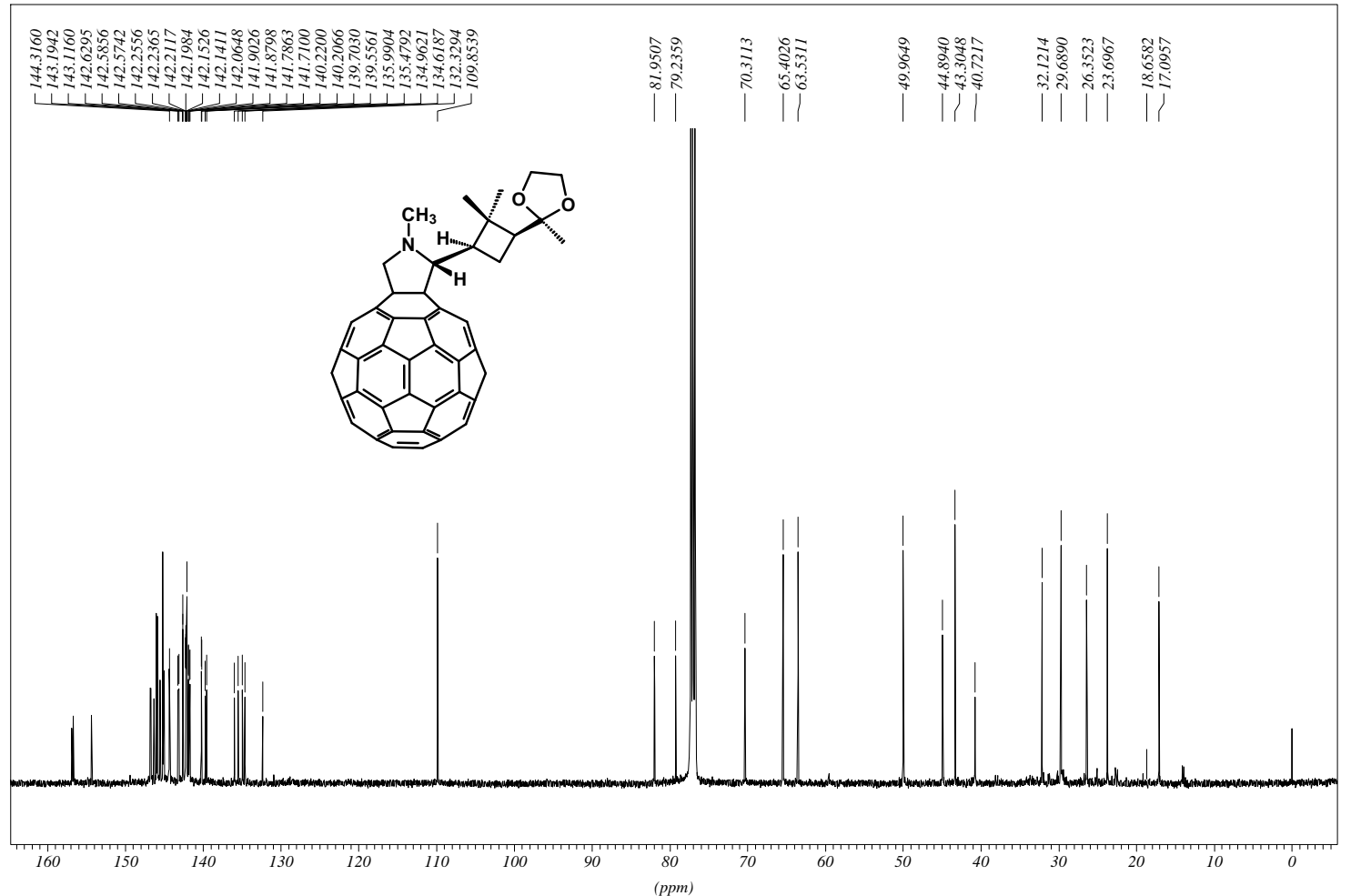




\section{References}

1. ADF2004. Baerends, E. J.; Autschbach, J. A.; Bérces, A.; Bo, C.; Boerrigter, P. M.; Cavallo, L.; Chong, D. P.; Deng, L.; Dickson, R. M.; Ellis, D. E.; Fan, L.; Fischer, T. H.; Fonseca Guerra, C.; van Gisbergen, S. J. A.; Groeneveld, J. A.; Gritsenko, O. V.; Grüning, M.; Harris, F. E.; van den Hoek, P.; Jacobsen, H.; van Kessel, G.; Kootstra, F.; van Lenthe, E.; Osinga, V. P.; Patchkovskii, S.; Philipsen, P. H. T.; Post, D.; Pye, C. C.; Ravenek, W.; Ros, P.; Schipper, P. R. T.; Schreckenbach, G.; Snijders, J. G.; Solà, M.; Swart, M.; Swerhone, D.; te Velde, G.; Vernooijs, P.; Versluis, L.; Visser, O.; van Wezenbeek, E.; Wiesenekker, G.; Wolff, S. K.; Woo, T. K.; Ziegler, T. Vrije Universiteit Amsterdam: Amsterdam, The Netherlands, 2004.

2. Baerends, E. J.; Ellis, D. E.; Ros, P. Chem. Phys. 1973, 2, 41.

3. te Velde, G.; Bickelhaupt, F. M.; Baerends, E. J.; Fonseca Guerra, C.; Van Gisbergen, S. J. A.; Snijders, G. J.; Ziegler, T. J. Comput. Chem. 2001, 22, 931.

4. te Velde, G.; Baerends, E. J. J. Comp. Phys. 1992, 99, 84.

5. Becke, A. D. Phys. Rev. A 1988, 38, 3098.

6. Perdew, J. P. Phys. Rev. B 1986, 33, 8822.

7. Snijders, G. J.; Baerends, E. J.; Vernooijs, P. At. Nucl. Data Tables 1982, 26, 483.

8. Vernooijs, P.; Baerends, E. J. Slater Type Basis Functions for the Whole Periodic System. Internal Report, Vrije Universiteit of Amsterdam, The Netherlands, 1981.

9. (a) Autschbach, J.; Ziegler, T. J. Chem. Phys. 2000, 113, 936. (b) Autschbach, J.; Ziegler, T. J. Chem. Phys. 2000, 113, 9410. (c) Autschbach, J.; Ziegler, T. J. Am. Chem. Soc. 2001, 123, 3341. (d) Autschbach, J.; Ziegler, J. Am. Chem. Soc. 2001, 123, 5320.

10. Krijn, J.; Baerends, E. J. Fit Functions in the HFS Method. Internal Report (in Dutch), Vrije Universiteit of Amsterdam, The Netherlands, 1984. 